\title{
Research on 3D Reconstruction of Facial Images based on Computer Vision
}

\author{
Jin Sun ${ }^{\text {a, }}$, Yuan Liu ${ }^{\text {a }}$, XinlongZhu ${ }^{\text {a }}$, Li Zeng ${ }^{a}$, Wangqin Zhang ${ }^{\text {a }}$, Xuelong $\mathrm{Hu}^{\text {a }}$, Fang Chen ${ }^{\mathrm{a}}$,Lifeng Zhang ${ }^{\mathrm{b}}$ \\ ${ }^{\mathrm{a}}$ College of Mechanical Engineering, Yangzhou University, Yangzhou, China \\ ${ }^{\mathrm{b}}$ Department of Electrical Engineering and Electronics, Kyushu Institute of Technology, Fukuoka, Japan \\ *Corresponding Author: sunjin1001@126.com
}

\begin{abstract}
The purpose of this paper is to describe how to reconstruct a 3D model based on a single front facial image. With the use of computer vision library named OpenCV and graphics library OpenGL, a 3D facial reconstruction software was developed in the environment of visual studio 2008. Firstly, the facial images in the format of bmp were read by this software. Then the key features in the face image were extracted. Finally, a personalized 3D face model was successfully reconstructed. The whole process only needs a single front face image which contains a neutral expression and uniform illumination conditions. In a word, the operation is simple and the speed is fast.
\end{abstract}

Keywords: computer vision, single-image, OpenCV, face model, 3D reconstruction.

\section{Introduction}

Face is a very complex physical structure and the most important part to identify a person. Since the 1970s, 3D facial reconstruction technique has been widely developed (1). Currently, 3D facial reconstruction has been initially applied to the video phone, a plurality of video conferencing, video production, computer games, virtual reality, medical research and other fields, and with the development of technology, 3D facial reconstruction will get deeper and broader applications in all respects. With the solve of this problem, it can provide an important reference to similar problems, such as face recognition, and it has an important role in promoting the new technology research and application. For 3D face image reconstruction technique based on the study, it can be generally divided into two methods: one is based on geometric images and the other is based on information. The 3D reconstruction method based on single image is to extract the target through a single image geometry and color and other information and to obtain 3D information of the target by using some known conditions.

\section{3D Reconstruction}

\subsection{Concept of 3D Reconstruction}

$3 \mathrm{D}$ reconstruction is defined according to one or more single view or multi-view image reconstruction of 3D model and the process of extracting or generating the corresponding characteristic information. It accurately reconstruct a 3D geometric model information based on the data and photo-realistic real scene.

\subsection{Research Progress}

In 2010, Zhu Qing sheng ${ }^{(2)}$ designed a simple plurality of images based on easy operation and low distortion 3D reconstruction system. In the feature match, we use SIFT feature descriptor to describe the feature point and propose the pole constraint to remove mismatch ,the match effect is good; in the process of obtaining the 3D coordinates, we select the most similar to the two-dimensional local information point for the most advantages. Reconstruction works well, modeling realistic and the degree of automation is high. But the number of feature point matching needs to be improved, display and camera calibration requires further study. In 2010 ,Deng Qiu ping ${ }^{(3)}$ of Shanghai Jiao tong University proposed a new statistical model, combining two-dimensional and three-dimensional information, the use of thin plate splines function effectively establishes the corresponding point of relations, and the establishment of a statistical model BJUT-3D three-dimensional human face database. But it needs to improve the face alignment accuracy to ensure that texture mapping does not produce errors. In 2011, Yu Chang ${ }^{(4)}$ of Xiangtan University use the left and right oblique side of the photo as input, through positive training methods $2 \mathrm{D}$ photos 3D face model to generate a three-dimensional 
human face which has a texture information, in the purpose to improve the traditional method which has a poor image mosaic effect and exist a model depth missing drawbacks. Adult students face high authenticity, but the texture information needs to be increased. In 2011, Chen Ting ${ }^{(5)}$ generated face model for geometry modifications, to give a specific face model. Where in the non-adjustment feature points by kriging interpolation function ${ }^{(6)}$ to achieve, Kriging interpolation method is a smooth interpolation method, the data points for a long time, the results of its interpolated credible higher degree of equations to be solved once in each non-feature point, the large amount of calculation.In 2011 Liu du $\operatorname{Jin}^{(7)}$ introduced chaotic mutation thought to solve APSO algorithm premature question, instead of the traditional back-propagation neural network (BPNN) gradient descent algorithm. It improves the feature of the traditional method which is time-consuming, difficult convergence, low precision, but in pose, illumination and expression changes in three-dimensional facial reconstruction effect. In 2012, Zhang $\mathrm{Xu}^{(8)}$ extracted hierarchical feature of 2D face image.He used feature vectors to reconstruct face parametric surfaces and reconstructed face in three-dimensional surface to resolve the problem by light and other environmental impacts and the two-dimensional image limit the amount of information caused by erroneous recognition of the problem, the accuracy rate of it is $98.6 \%$. In 2013 , Xiong Ping ${ }^{(9)}$ of Central South University combined the level set method ${ }^{(10)}$ with SFS algorithms to build a $3 \mathrm{D}$ face model, improving traditional reconstruction algorithms cannot accurately locate the face shape problems, the algorithm complexity is low, the computation time is short, true shape of the face can be guaranteed. But the photos must be input frontal face photo, a deflection angle cannot be allowed.In 2012, Cheng Wen-Po and Tien-ruey Hsiang $^{(11)}$ proposed the establishment of the tensor-based three-dimensional face model from a color image and obtained the depth information from based CCA map between the tensor model. Establish tensor model must takes the multi-color factor into account.Zhang Jian ${ }^{(12)}$ et al proposed a rule to restored facial expressions accurately from the general shape of a single image. Establish the equation contains expressions depending on the specific parameters of the camera projection model, so the unknown parameters can be easily solved by Newton's method.

\subsection{The 3D Modeling Method of Face}

1) Spline pseudo-muscle model. Pseudo muscle model does not involve a face inside the complex physical structure, howerver, a similar manner was used on the face muscles for the implement of the grid deformation, and the deformation occured only on the local grid. Free form model belongs to the pseudo-spline model muscle. Specifically, it is a deformable object embedded in an elastic three dimensional grid of points by the control of an imaginary control box. While the control box was squeezed, bended and twisted, the object embedded in the control box were deformed correspondingly.

2) Statistical Model. Based on statistical modeling approach, Blanz, Volker, and T. Vetter ${ }^{(13)}$ firstly established a large face database for statistical model, then used the face database of face images to approximate the specific face in the photo by linear combination. This method was done automatically, and highly adaptable for different photos. The advantage of this method is that the resulting model is realistic, with no need of any human intervention, However, its drawback is that a large face database need to be established in advance. Because the face database may be very big, but it is still impossible to include all of the world face shape, so the simulated human face played a limited role to a certain extent.

3) Facial Action Coding System. Essa et.al ${ }^{(14)}$ used modular eigenspace way to deal with the problem of adaptation aspects. The method may select facial features on the feature point position in the image automatically. Using these feature points may control the image distortion through deformation, you can make a personalized face image with the generic face model match. Vertex deformation can be extracted from the image out to save, and then prepare for further detailed work. By free deformation, deformation of random noise, site editor, or digital organisms to control the existing model to generate new specific face model.

\section{The 3D Date Acquisition of Face}

\subsection{Retrieving Data from the three-dimensional laser scanner}

Three-dimensional laser scanner technology, also known as real replication technology. Using laser ranging principle, the original data as a point cloud data and it can be used directly after simple treatment, the process after the data is omitted. Using non-contact manner, to adapt to a variety of complex environments, with fast, simple and accurate. By using three-dimensional laser scanner to obtain facial feature 
points $\mathrm{X}, \mathrm{Y}, \mathrm{Z}$ coordinates and the letter $\mathrm{R}, \mathrm{G}, \mathrm{B}$ color information, and additional information such as the rate of anti-color, face data is more comprehensive and accurate. The current production of laser scanner manufacturers are US companies FARO, USA Cyberware, Switzerland Leica Company and so on,as shown in Fig.1.And the classification of 3D laser scanners can be seen in Table.1.

\subsection{Retrieving Data from the face database}

Face database is an important basis for research and development of model training to human face, the establishment of a large scale, covering a wide variety of face database is of great significance. In general, the face database is divided into two, namely face database and face graphics library. Face image library includes a set of different face angle photo,as shown in Fig.2. Although without depth information, we can read the depth information according to the prior art of the number of sheets with many and different angles. The graphics library of facial features used physical means such as laser scanners to obtain the data of facial features. Currently authoritative and commonly face database are:

1) FERET face database contains over 14,051 Face Image ${ }^{(15)}$.They have different pose and lighting conditions , and all have gradation processing, most of them are Westerners, and each face have a single image change.

2) The PIE face database of Carnegie Mellon University contains more than 41,368 different pose, illumination and expression facial image of the 68 volunteers $^{(16)}$

3) The large face databases of CAS CAS-PEAL ${ }^{(17)}$, including 1040 Chinese man's head shoulder head, covering some change of conditions such as posture, facial expressions, and other accessories and lighting, some people face images with the background, distance and time span changes, which number of the total is up to 99450.

4) The China large facial database of BJUT- $3 \mathrm{D}^{(18)}$ from Beijing University of Technology contains 500 three dimensional face about Chinese people, men and women face each accounted for half. People all maintain a neutral facial expression and do not wear glasses and ornaments in the process of scan.

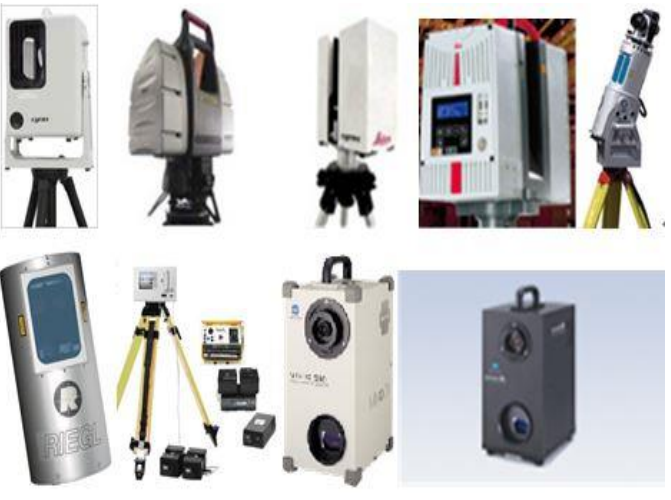

Fig. 1. Three dimensional laser scanner.

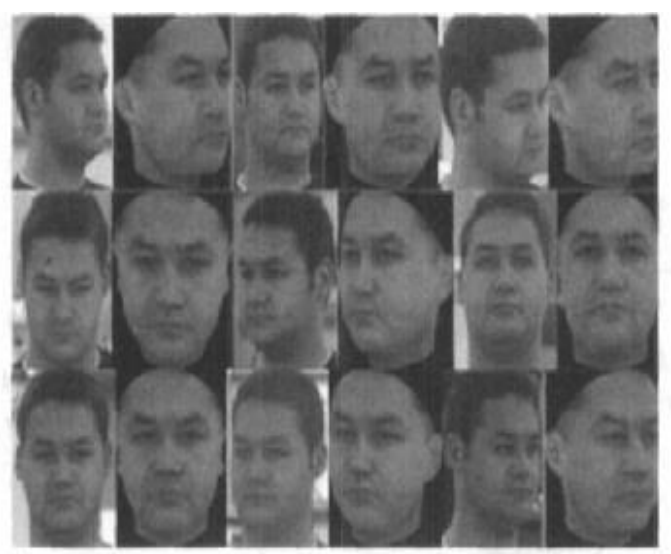

Fig. 2. 3D face database.

Table 1. Classification of 3D laser scanner.

\begin{tabular}{|c|c|c|c|c|}
\hline $\begin{array}{c}\text { Classification } \\
\text { index }\end{array}$ & \multicolumn{4}{|c|}{ Instrument type } \\
\hline Carrying platform & $\begin{array}{c}\text { Machine } \\
\text { used }\end{array}$ & $\begin{array}{c}\text { Vehicle } \\
\text { used }\end{array}$ & Fixed & Handheld \\
\hline Scanning distance & $\begin{array}{c}\text { Long } \\
\text { distance }\end{array}$ & $\begin{array}{c}\text { Medium } \\
\text { distance }\end{array}$ & $\begin{array}{c}\text { Short } \\
\text { distance }\end{array}$ & $\begin{array}{c}\text { Super } \\
\text { short } \\
\text { distance }\end{array}$ \\
\hline Scanning mode & \multicolumn{2}{|c|}{ Line scanning system } & \multicolumn{2}{|c|}{ Area scanning system } \\
\hline
\end{tabular}

\section{3D Facial Modeling System}

The workflow of 3D facial modeling system can be seen in Fig.3.

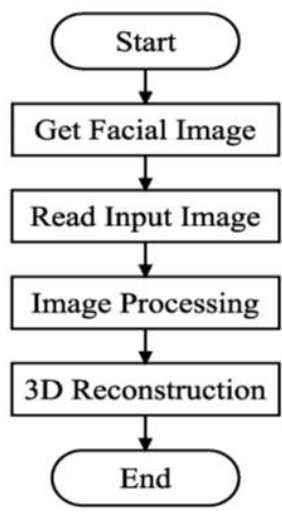

Fig. 3. The workflow of 3D facial modeling system. 


\subsection{Read the Photo of Face}

After obtaining the digital files of the facial photoes, the specified input file must be $24 \mathrm{bmp}$ image, and the non-bmp file must be transformed into bmp file format, and then be read into the software,as shown in Fig.4.

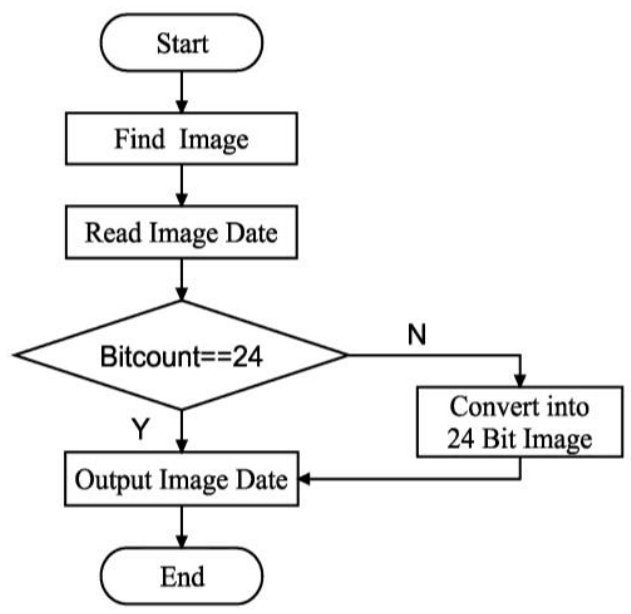

Fig. 4. The algorithm of face image reading.

\subsection{Facial Reconstruction}

Step 1: Window initializatiing which mainly consists the definition of pixel format, setting the pixel format and the creation of the $\mathrm{RC}$ file.

Step 2 : Setting the graphic display mode to determine the viewport work, and defining a calculation that contains the viewport and viewport orientation.

Step 3: Rendering three-dimensional graphics, including initialization and drawn scenes, the drawing about point and polygon.

Step 4 : Dealing with the aftermath, including undoes the scene and render environment, as well as rendering the environment handle deleted.

\subsection{Experimental Results}

3D face system interface operation is very simple. First, after the "File" option in the menu bar was clicked, the "Image File" option was selected,the icon of "Loading image file" poped on the screen of the software automatically.Then,after the icon of "Open"was clicked to select the photos you want to enter the face (previously converted into BMP format), the 3D face model will display according to the software input face photo automatically. By moving the mouse and the keyboard arrow keys to generate the model control, in the purpose of achieving rotation, zoom in, zoom and other operations. The experimental results can be seen in Fig.5 and Fig.6.

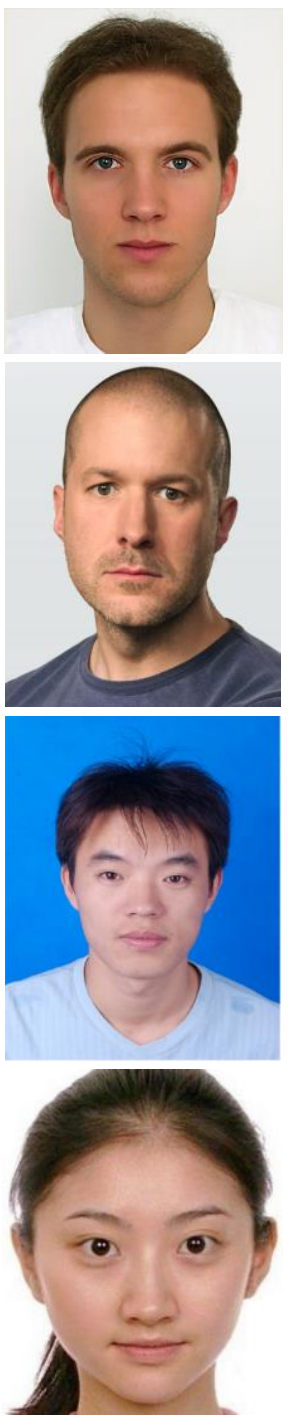

Fig. 5. Test picture.
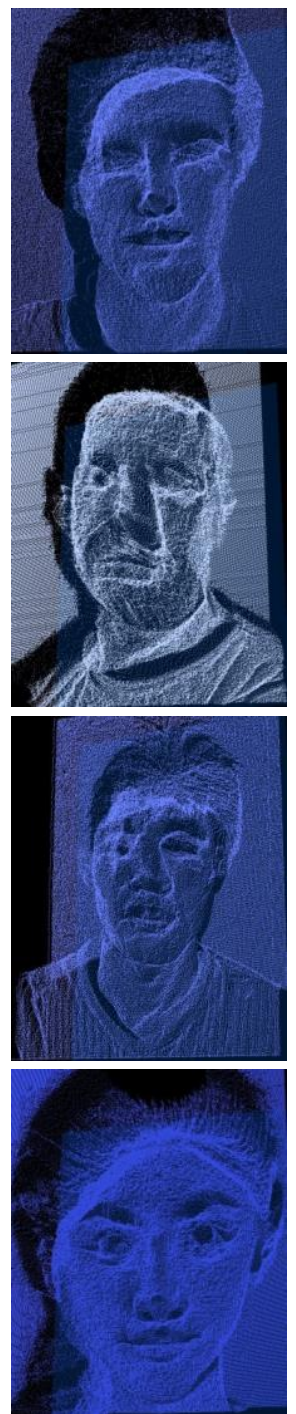

Fig. 6. Experiment result.

\section{Copyright}

Papers submitted to ICISIP must describe original and unpublished researches. They should not have been submitted to other conferences or journals. Any paper which is under review elsewhere will not be considered for publication. Once an eligible paper is accepted, it will be published under a Creative Commons Attribution 3.0 (CC BY 3.0) License.

\section{Conclusions}

In this paper, three-dimensional model reconstruction method of single frontal face photo is studied. A three-dimensional facial reconstruction algorithm is 
presented.Specific research can be divided into the following sections:

1) The Status and results of three-dimensional facial reconstruction are discussed in detail, focusing on the face reconstruction based on single photo;

2) A new method of facial reconstruction is proposed;

3) A facial reconstruction software is developed, the software automatically generates a three-dimensional model with the help of human input face photo.

As soon as the technology itself is concerned, there are still many areas for improvement, such as the lack of texture mapping information, such as missing for three-dimensional data preservation, this is where the need for further research, will further improve in the future work.

\section{Acknowledgment}

This work was supported by National Natural Science Foundation of China(Grant No. 51475409), Jiangsu Province Basic Research Program Natural Science Foundation (Grant No. BK20141277), Yangzhou city Yangzhou University of Science and Technology Cooperation Program Funds(No. YZ2016244), 2016 Six talent peaks project in Jiangsu Province (No. JXQC-030).

\section{References}

(1) Parke, Frederick I: "Computer generated animation of faces", Proceedings of the ACM National Conference, pp. 451-457,1972

(2) Zhu Qing-sheng, Luo Da-jiang, Ge Liang, Liu Jin-feng: "Multiple-image-based 3D reconstruction", Computer Engineering and Design, Vol. 31, No. 10, pp. 2351-2357, 2010

(3) Deng Qiuping: "3D Face reconstruction based on a single image and statistical model", Shanghai Jiao Tong University,2010

(4) Yu Chang,Chen Yi-qiang,Liu Jun-fa,Tang Xiao-qing : "Method of reconstructing 3D human head based on two half-frontal photos", Application research of computers, Vol. 28, No. 10, pp. 3944-3946, 2011

(5) Chen Ting, Alifu Kuerban: "Method of individual 3D face reconstruction based on photograph" , Computer Technology and Development, Vol. 21, No. 6, pp. 43-45,2011

(6) Jin Guodong, Liu Yancong,Niu Wenjie: "Comparison between inverse distance weighting method and kriging", Journal of Changchun University of
Technology, Vol. 24, No. 3, pp. 53-57,2003

(7) Liu Du jin,Sun Shuxia,Li Siming: "Three-dimensional facial reconstruction method based on improved BPNN for single photo", Computer Simulation, Vol. 28, No.9, pp. 275-278, 2011

(8) Zhang Xu: "Recognition Algorithm Based on 2d Face Images", Computer Simulation, Vol. 29, No. 3, pp. 330-333, 2012

(9) Xiong Ping,Lu Ye: "Modified 3D facial reconstruction algorithm based on a single photo", Journal of Computer Applications, Vol. 33, No. 8, pp. 2359-2361, 2013

(10) Wang S Y, Lim K M,Khoo B C: “An extended level set method for shape and topology optimization", Journal of Computational Physics, Vol. 221, No. 1, pp. 395-421, 2007

(11)Cheng Wen Po, Tien-ruey Hsiang: "Tensor-based 3D color face reconstruction using a single image", International Conference on Control Automation Robotics \& Vision, pp. 883-888, 2013

(12) Zhang Jian, Sun Rui, Zhan Xiaosi: "Accurate human face pose recovery from single image through generic shape regularization", Signal Processing, Vol. 110, pp. 5-14, 2015

(13) Blanz, Volker, and T. Vetter: "Face recognition based on fitting a 3D morphable model", IEEE Transactions on Pattern Analysis \& Machine Intelligence, Vol. 25, No. 9, pp. 1063-1074, 2003

(14) Essa, Irfan A, A. P. Pentland: "Coding, analysis, interpretation, and recognition of facial expressions", IEEE Transactions on Pattern Analysis \& Machine Intelligence, Vol. 19, No. 7, pp. 757-763, 2002

(15) Phillips, P. Jonathon: "The FERET database and evaluation procedure for face-recognition algorithms", Image \& Vision Computing, Vol. 16, No. 5, pp. 295-306, 1998

(16) Sim, Terence, S. Baker, M. Bsat.: "The CMU pose, illumination, expression (PIE) database", IEEE International Conference on Automatic Face and Gesture Recognition, Vol. 25, No. 12, pp. 46-51, 2002

(17)Zhang Xiaohua,Shan Shiguang,Cao Bo,Gao Wen,Zhou Delong,Zhao Debin: "Principal warps: CAS-PEAL:A large-Scale chinese face database and some primary evaluations", Journal of Computer-aided Design \&Computer Graphics, Vol. 17, No.1, pp. 9-17, 2005

(18) Yin Baocai,Sun Yanfeng, Wang Chengzhang, Ge Yun: "BJUT-3D large scale 3D face database and 
information processing", Journal of computer research

and development, Vol. 46, No. 6, pp. 1009-1018, 2009 